\title{
ANGEBORENE STOFFWECHSELKRANK- HEITEN BEIM ERWACHSENEN: WIE SIEHT ES IN DER SCHWEIZ AUS?
}

Christel Tran, Andrea Superti-Furga, Michel Hochuli

Übersetzer: Rudolf Schläpfer

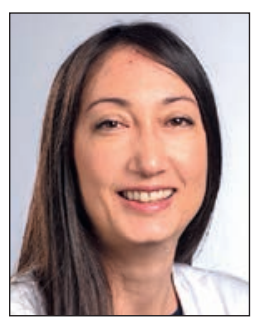

Christel Tran

\section{Einführung}

Angeborene Stoffwechselkrankheiten (ASK) sind die Folge eines genetischen Defektes von Enzymen oder regulierenden Eiweissen, die in einige metabolische Prozesse eingreifen.

Auch wenn jede einzelne selten ist, umfasst die heutige Nosologie über 1000 Stoffwechselkrankheiten ${ }^{1)}$. Die kollektive Prävalenz wird heute auf über 1:800 Individuen geschätzt ${ }^{2}$. Bis vor kurzem war die Betreuung der ASK vor allem pädiatrisch, auf hergebrachten Strukturen beruhend, in welchen Diagnostik und/oder Screening durch Kinderärzte gewährleistet wurden.

Diagnostik und Betreuung der ASK haben im Verlaufe der letzten 20 Jahre jedoch bedeutende Fortschritte gemacht, was eine deutliche Verbesserung der Prognose eines Teils dieser Krankheiten zur Folge hat. Verbesserte Screeningprogramme, vermehrte Sensibilisierung für diese Krankheiten, verfeinerte diagnostische Tests und wirksamere therapeutische Massnahmen haben nicht nur die Lebenserwartung der Patienten verlängert und die Prognose verbessert, sondern führen auch dazu, dass sich später manifestierende Formen bei zunehmenden Zahlen an erwachsenen Patienten nachgewiesen werden ${ }^{3,4)}$.

Eine kürzlich durch das europäische Referenznetzwerk für ASK durchgeführte Untersuchung ergab, dass heute 50\% der Patienten mit einer ASK erwachsen sind (wissenschaftlicher Bericht, Jahresversammlung 2018 des Verwaltungsrates, MetabERN). Da viele dieser Patienten nun ein fortgeschrittenes Alter erreichen können, ist die Ausbildung der Ärzte in diesem Gebiet und die Schaffung spezialisierter Dienste für ASK beim Erwachsenen eine Notwendigkeit geworden. Diese günstigeren klinischen Verläufe von Patienten mit ASK gehen mit einer Diversifizierung des klinischen Spektrums und dem Auftreten von Komplikationen und Problemen einher, die im Kindesalter nicht angetroffen werden. Diese für das Erwachsenenalter spezifischen Faktoren der ASK stellen eine Herausforderung für die Erwachsenenmedizin dar, die die Langzeitbetreuung dieser Patienten übernehmen soll 5,6$)$.

Ziel dieses Artikels ist es, eine Übersicht des Netzwerkes zu geben, das sich um die ASK im Erwachsenenalter gebildet hat, und die Besonderheiten der Be- treuung dieser Patienten anhand einiger emblematischen Fällen zu beschreiben.

\section{Welche Herausforderungen erwartet die Erwachsenenmedizin? \\ Ärztliche Ausbildung}

In der Vergangenheit wurde die Medizin der ASK als eine pädiatrische Disziplin betrachtet, für die es keine spezifische Ausbildung in Erwachsenenmedizin gab. Leider gibt es in der Schweiz immer noch kein etabliertes Ausbildungscurriculum. Die Schwierigkeit besteht darin, dass es sich um eine neue Disziplin handelt, die verschiedene Fachrichtungen betrifft, wie Genetik, Biochemie, Neurologie, Stoffwechsel, Ernährung, Innere Medizin u.a.m.

Es ist deshalb nicht einfach festzulegen, wie das ideale Curriculum für eine eigenständige Fachrichtung aussehen soll. Eine kürzlich weltweit durchgeführte Umfrage ergab, dass die meisten Zentren, die weltweit Patienten mit ASK betreuen, finden, die aktuellen Ausbildungsmöglichkeiten in diesem Bereich seien ungenügend ${ }^{7}$ ). Um dieser auf der ganzen Welt unumgänglich gewordenen Nachfrage gerecht zu werden, laufen in mehreren Ländern spezifische Ausbildungsprogramme ${ }^{8)}$.

In der Schweiz integriert die Weiterbildung zum Facharzttitel FMH Endokrinologie/Diabetologie Kenntnisse zur Pathophysiologie der wichtigsten ASK im Erwachsenenalter.

\section{Klinische Betreuung}

Dank den Fortschritten in der pädiatrischen Betreuung können Patienten mit im Kindesalter diagnostizierten Stoffwechselkrankheiten nun ein fortgeschrittenes Alter erreichen. Zudem werden bei einer signifikanten Anzahl Menschen Stoffwechselkrankheiten heute im Erwachsenenalter diagnostiziert. Die Ausbildung der Erwachsenenärzte sowie die Schaffung von spezialisierten Zentren für ASK sind eine Notwendigkeit geworden. Diese günstigeren klinischen Verläufe von Patienten mit ASK gehen mit einer Diversifizierung des klinischen Spektrums und dem Auftreten von Komplikationen und Problemen einher, die im Kindesalter nicht angetroffen werden.

Diese für das Erwachsenenalter spezifischen Faktoren der ASK stellen für die Ärzte, die nach der Transition die Betreuung übernehmen eine grosse Heraus- 


\section{Fortbildung}

forderung dar, und werfen zudem mehrere Fragestellungen auf: 1) Zahlreiche ASK sind multisystemische Krankheiten die eine interdisziplinäre Betreuung und Koordination unter den verschiedenen Fachpersonen erfordern, 2) Vorbeugung von Komplikationen, 3) weitere altersbedingte Probleme/Krankheiten des Patienten für welche therapeutische Massnahmen unter Berücksichtigung der ASK getroffen werden müssen, und schliesslich 4) spezifische Situationen des Erwachsenenalters, z.B. Schwangerschaft.

In einer kürzlich in zwei Spitalzentren der Schweiz (Lausanne und Genf) durchgeführten retrospektiven Studie wurde bei 126 erwachsenen Patienten mit ASK der Verlauf über vier Jahre nach der Transition untersucht. Über 50\% der Patienten mit einer bestätigten Diagnose ASK entwickelten Komplikationen im Zusammenhang mit der Stoffwechselkrankheit. Davon mussten $19 \%$ auf Grund einer metabolischen Dekompensation notfallmässig hospitalisiert werden und benötigten eine spezifische Therapie, bei $69 \%$ verschlimmerten sich bekannte vorbestehende Komplikationen und 13\% entwickelten zuvor unbekannte Komplikationen ${ }^{9}$.

Diese Komplikationen traten auf, obwohl die meisten dieser Patienten regelmässige Kontrolluntersuchungen in einer Klinik hatten, und bekunden die Wichtigkeit eines koordinierten Betreuungsnetzes sowie der Möglichkeit, ohne Verzug die notwendigen therapeutischen Schritte einleiten zu können - obwohl die Prognose quoad vitam sich stark gebessert hat, weist die Grundkrankheit eine signifikante Morbidität auf. Für Patienten die (noch) keine Komplikationen zeigen oder an funktionellen Einschränkungen leiden, ist eine engmaschige Betreuung durch den Bezugsarzt wichtig, damit möglichst wenige Patienten einer fachärztlichen Behandlung entgehen.

\section{Medizinisch-wirtschaftliche und ethische Aspekte}

Gewisse ASK benötigen Therapien z.B. durch Enzymersatztherapie, die Verabreichung von Enzym-Kofaktoren oder eine Ernährungstherapie mit spezifischen diätetischen Produkten, deren Kosten erheblich sein können.

In der Schweiz deckt die Invalidenversicherung (IV) die Kosten dieser Therapien nur bis zum 20. Altersjahr, die Kostenübernahme für ärztliche Leistungen bei Geburtsgebrechen endet damit. Wohl sieht der Gesetzgeber das Prinzip der Kontinuität der Kostenübernahme beim Übergang von IV zur obligatorischen Grundversicherung vor, doch gibt es rechtliche Grauzonen, die zu Schwierigkeiten führen können. Es gibt zum Beispiel keine klaren Regeln zur Kostenübernahme einer spezifischen Behandlung, die erst im Erwachsenenalter beginnt, abgeändert oder ergänzt werden muss, ebenso wenn ein Patient aus dem Ausland zugezogen ist und zuvor nicht bei der IV angemeldet war.

Die Krankenkassen können zudem die Kostenübernahme einer von der IV bezahlten Behandlung verweigern, falls sie noch nicht in der Spezialitätenliste (SL) oder der Geburtsgebrechenmedikamentenliste (GGML) aufgeführt ist. Diese Situationen werden sowohl von den Patienten als auch den Ärzten als unangenehm empfunden. Die Patienten fühlen sich isoliert und durch das Gesundheitssystem verlassen, empfinden den Verlust der pädiatrischen Betreuung. Die Ärzte ihrerseits müssen unzählige administrative Schritte unternehmen, um die Kostenübernahme für Behandlungen zu erreichen.

Im September 2019 haben die Mitgliedländer der Vereinten Nationen, die Schweiz inbegriffen, eine Erklärung zur Universellen Gesundheitsversorgung angenommen (Interpellation No. 20.4083), mit welcher sie sich verpflichten, die Betreuung seltener Krankheiten zu verbessern. Gemäss der WHO besteht die universelle Gesundheitsversorgung darin, dass alle Menschen Zugang zur ärztlichen und pflegerischen Betreuung haben, die sie benötigen, ohne dass der Nutzniesser dadurch in finanzielle Schwierigkeiten gerät. Auf Vorschlag von ProRaris (Allianz Seltener Krankheiten Schweiz) wurde die Interpellation im September 2020 dem Nationalrat unterbreitet.

Die erwähnten Probleme werden im Prinzip anerkannt und gehören zum nationalen Konzept Seltene Krankheiten des Bundesamtes für Gesundheit. Der Bundesrat hat im Februar 2021 einen Bericht unter dem Titel «Gesetzliche Grundlage für die Sicherstellung der Versorgung im Bereich der seltenen Krankheiten» erstellt, in welchem er präzisiert, welche gesetzliche Änderungen und finanziellen Mittel notwendig sind, um die Versorgung in diesem Bereich zu verbessern.

\section{Das schweizerische Netzwerk}

Um optimal auf die Bedürfnisse der erwachsenen $\mathrm{Pa}$ tienten mit ASK eingehen zu können, wurden in verschiedenen Spitalzentren der Schweiz spezialisierte Sprechstunden geschaffen. Diese Konsultationen finden in enger Zusammenarbeit und intensiven Austauschen mit pädiatrischen ASK-Fachärzten statt, und sind in der Webseite https://inbornerrors.ch, Kapitel Sprechstunde für Erwachsene erfasst. Eine Untergruppe (The Swiss Adult Metabolic Group) der SGIEM (Swiss Group of Inborn Errors of metabolism, www. inbornerrors.ch) umfasst die Vertreter dieser Spezialsprechstunden. Die Treffen ermöglichen es, sich zu verschiedenen Aspekten austauschen zu können, z.B. 1) klinische (Betreuung der Patienten, z.B komplexe Fälle, neue Therapien), 2) akademische (z.B. laufende Studien, Weiterbildung), 3) sozioökonomische (z.B. Kostenübernahme von Therapien, Antragstellung bei den Kostenträgern). Diese Treffen dienen ebenfalls dazu, enge Kontakte unter den verschiedenen Beteiligten aufrecht zu erhalten.

\section{Fallstudien}

\section{Phenylketonurie und Schwangerschaft}

Es handelt sich um eine 28-jährige Frau, bei welcher eine Phenylketonurie (PKU) bei der Geburt in Belgien diagnostiziert wurde. Während ihrer Kindheit musste sie eine spezifische Diät befolgen. Dank der gut eingehaltenen Diät waren die Serumphenylalaninwerte 


\section{Fortbildung}

immer im Zielbereich, die psychomotorische Entwicklung normal und der Schulbesuch problemlos. Als Erwachsene beschliesst sie, die ärztliche Betreuung zu unterbrechen. Da sie keinen Unterschied ihres Allgemeinzustandes mit oder ohne Diät feststellt, gibt sie nach und nach die Ernährungsvorschriften auf. Sie lernt ihren Partner mit 27 Jahren kennen und wird ungewollt schwanger. Im selben Jahr zieht das Paar aus beruflichen Gründen in die Schweiz. Sie weiss, dass während einer Schwangerschaft das Serumphenylalanin überwacht werden muss, um fötale Komplikationen zu verhindern. Da sie seit mehreren Jahren keine Diät mehr eingehalten hat, konsultiert sie die Swiss Adult Metabolic Group ihrer Region.

Die PKU (OMIM \#261600) wird bei der Geburt gescreent. Die Inzidenz in der Schweiz beträgt 1:8000 (www.neoscreening.ch). Die Hyperphenylalaninämie ist bedingt durch einen Mangel an Phenylalaninhydroxylase, deren Funktion darin besteht, Phenylalanin in Gegenwart eines Kofaktors (BH4) zu Tyrosin zu hydroxylieren. Es kommt zu einer Anhäufung von Phenylalanin und einem Mangel an Tyrosin, einer Vorstufe von Neurotransmittern. Unbehandelt verursacht die Hyperphenylalaninämie beim Kind einen schweren Entwicklungsrückstand. Die Behandlung besteht in einer kontrollierten Zufuhr von Phenylalanin (an natürlichen Eiweissen arme «PKU-Diät»), um den Plasmaspiegel im altersgerechten Normbereich zu halten und eine normale Hirnentwicklung zu ermöglichen ${ }^{10)}$. Die Beibehaltung der PKU-Diät im Erwachsenenalter, mit einem Plasmaspiegelwert $<600 \mu \mathrm{mol} /$ l, hat zum Ziel, eventuell spät auftretenden neuropsychologischen Störungen vorzubeugen ${ }^{11)}$. Diese Diät ist jedoch anspruchsvoll und die Vorteile für die Erwachsenen nicht immer klar ersichtlich, weshalb eine beträchtliche Anzahl Patienten darauf verzichtet. Hingegen ist die Betreuung der Schwangerschaft bei PKU-Patienten wegen des Risikos einer PKU-bedingten Embryopathie (Mikrozephalie, geistiger Entwicklungsrückstand, angeborene Herzmissbildung) von grosser Bedeutung. Um diese Risiken zu vermindern, muss die Diät vor der Konzeption begonnen und bis zur Geburt beibehalten werden, mit einem Zielwert für Plasmaphenylalanin gemäss schweizerischen Empfehlungen $<300$ $\mu \mathrm{mol} / \mathrm{I}^{12)}$.

\section{Katamnese}

Diese Patientin befand sich in der 12. SSW als sie das Zentrum konsultierte, und ihr Phenylalaninspiegel betrug $1250 \mu \mathrm{mol} / \mathrm{l}$. Angesichts des Risikos einer Embryopathie zog es das Ehepaar vor, die Schwangerschaft zu unterbrechen. Dies hatte schwerwiegende Folgen für die junge Frau, die eine schwere Depression erlitt. Für diese Frau, die ihre Behandlung mit dem Erreichen des Erwachsenenalters unterbrach, wäre eine regelmässige Betreuung und Einhaltung der Diät bevor sie schwanger wurde, von Vorteil gewesen.

\section{Persistierende Thombozytopenie mit mässiger Splenomegalie}

Ein 48-jähriger Mann sucht seinen Hausarzt wegen wiederholtem Nasenbluten auf. Das Blutbild zeigt eine isolierte Thrombopenie von 80G/I und der Ultraschall des Abdomens eine leichte Milzvergrösserung. Die MRT bestätigt die Splenomegalie bei normaler Lebergrösse, und weist eine Infiltration des Knochenmarks nach. Es wird ein Morbus Gaucher vermutet. Die darauf gemessene Enzymaktivität der ß-Glukozerebrosidase in den Leukozyten erweist sich als erniedrigt. Die molekulargenetische Untersuchung des Gens GBA offenbart zwei pathogene, komposit heterozygote Varianten, die den Morbus Gaucher Typ 1 bestätigen (OMIM \# 230800). Die Behandlung des Mo. Gaucher beruht in erster Linie auf der zweiwöchentlichen intravenösen Enzymsubstitution, einer Behandlung, die entsprechende Infrastrukturen erfordert ${ }^{13)}$. Diese Behandlung ist teuer und die Kosten werden von der Krankenkasse übernommen, wenn die Diagnose bestätigt ist und Krankheitssymptome vorliegen. Es besteht jedoch kein formeller Konsens betreffend Beginn der Behandlung bei erwachsenen Mo. Gaucher Patienten mit nur diskreten bzw. fehlenden Symptomen ${ }^{14)}$.

\section{Katamnese}

In der interdisziplinären Fachgruppe für Stoffwechselkrankheiten wurde, gemeinsam mit dem Patienten beschlossen, die Behandlung auf Grund des Knochenmarkbefalls und der Thrombopenie einzuleiten, mit dem Ziel, den Gesundheitszustand zu erhalten und Komplikationen der Zielorgane zu vermeiden (oder zu begrenzen). Es wurde ein Behandlungsplan festgelegt und der Patient in der spezialisierten Sprechstunde weiterbetreut, wo auch die notwendigen Laborkontrollen koordiniert werden.

\section{Muskelschmerzen und anstrengungsbedingte Rhabdomyolyse}

45-jähriger Mann mit bekannter, im Alter von 41 Jahren anlässlich einer Konsultation beim Neurologen wegen Muskelschmerzen diagnostizierter Glykogenose Typ V (Morbus McArdle, OMIM \# 232600). Die Muskelbiopsie zeigte einen Myophosphorylase-Mange ${ }^{15)}$. Es erfolgte keine regelmässige ärztliche Betreuung, bis inn sein neuer Hausarzt an die interdisziplinäre Fachgruppe für ASK des Erwachsenen verwies, um bezüglich weiterem Vorgehen beraten zu werden.

Der Patient erinnerte sich daran, dass er als Kind, als er seinem Vater auf dem Bauernhof half, wegen Schmerzen bei körperlicher Anstrengung häufig Pausen einlegen musste, um sich zu erholen. Vor sieben Jahren - mehrere Jahre vor Diagnosestellung - erlebte er eine Episode schwerer Myalgie, die inn während mehreren Tagen ans Bett fesselte. Der Patient zeigt typische Glykogenose Typ V-Symptome, in Form rasch auftretender Muskelschmerzen, z.B. wenn er etwas schneller geht oder eine Steigung emporgeht. Er erlebt auch das typische Second-Wind-Phenomenon, wobei ein Pausieren nach dem ersten Auftreten von Symptomen zu einer anschliessend guten und beschwerdearmen Muskelbelastbarkeit führt. Die Einnahme von Kohlehydraten vor einer geplanten Anstrengung verbessern die Leistungsfähigkeit.

Der Patient hatte auch einige Sitzungen Physiotherapie gehabt, was jedoch zu keiner signifikanten 


\section{Fortbildung}

Verbesserung im Alltag führte, vor allem auch weil er keine spezifische an seine Krankheit angepasste Empfehlungen bekam. Beruflich muss er körperliche Arbeit leisten und erreicht rasch die Grenze der Belastbarkeit, was zu Konflikten im Arbeitsteam führte. Da er befürchtete, entlassen zu werden, fand keine Diskussion mit dem Arbeitgeber statt. Den letzten dunklen Urin beobachtete er nach einer grossen Anstrengung vor ein paar Monaten. Die Laboruntersuchungen ergaben kürzlich massiv erhöhte CK Werte bei erhaltener Nierenfunktion.

Im Verlaufe des Gesprächs wurde klar, dass der Patient kaum Kenntnisse der Pathophysiologie seiner Muskelkrankheit, der Trainingsmöglichkeiten und notwendigen Massnahmen hatte.

\section{Katamnese}

In Anschluss an die Konsultation bei der interdisziplinären Fachgruppe für ASK des Erwachsenen, wurde der Patient in Zusammenarbeit mit Physiotherapeuten und mit Hilfe von Informationsbroschüren instruiert, wie er körperliche Anstrengungen im täglichen Leben und am Arbeitsplatz dosieren und sie so seinen Fähigkeiten anpassen kann. Mit der Unterstützung der Sozialhilfe konnte die Situation am Arbeitsplatz entspannt werden.

Zusammenfassend illustrieren diese drei Fallbeispiele die Schwierigkeiten, die bei der Transition und durch fehlende Betreuung im Erwachsenenalter auftreten, die Einstellung gegenüber bestimmten therapeutischen Entscheiden und schliesslich die möglicherweise negativen Konsequenzen, wenn die Diagnose im Erwachsenenalter gestellt wird ohne von Informationen und koordinierter Betreuung durch Fachärzte gefolgt zu sein. Wir unterstreichen deshalb, wie wichtig es ist, eine regelmässige Betreuung dieser Patienten aufrecht zu erhalten, damit die seit der Geburt unternommenen Bemühungen und die damit erreichten Erfolge auch im Erwachsenenalter andauern. Wird die Diagnose ASK im Erwachsenenalter gestellt, ist es wichtig, mit einem für ASK spezialisierten Zentrum zusammenzuarbeiten. Eine angepasste Information und Betreuung können den Verlauf der Krankheit und die Lebensqualität des Patienten wesentlich beeinflussen.

\section{Referenzen}

1) Ferreira CR, van Karnebeek CDM, Vockley J, Blau N. A proposed nosology of inborn errors of metabolism. Genet Med 2019; 21(1): 102-106. e-pub ahead of print 2018/06/10; doi: 10.1038/ s41436-018-0022-8
2) Wilcox G. Impact of pregnancy on inborn errors of metabolism. Rev Endocr Metab Disord 2018; 19(1): 13-33. e-pub ahead of print 2018/09/11; doi: 10.1007/s11154-018-9455-2

3) Werkgroep I. [Adults with an inherited metabolic disorder: a rapidly growing population with unique challenges]. Nederlands tijdschrift voor geneeskunde 2014; 158: A7745. e-pub ahead of print 2014/08/07

4) Lee PJ. Growing older: the adult metabolic clinic. Journal of inherited metabolic disease 2002; 25(3): 252-260. e-pub ahead of print 2002/07/26; doi: 10.1023/a:1015602601091

5) Enns GM, Packman W. The adolescent with an inborn error of metabolism: medical issues and transition to adulthood. Adolesc Med 2002; 13(2): 315-329, vii. e-pub ahead of print 2002/05/03;

6) Schwarz M, Wendel U. [Inborn errors of metabolism (IEM) in adults. A new challenge to internal medicine]. Med Klin (Munich) 2005; 100(9): 547-552. e-pub ahead of print 2005/09/20; doi: 10.1007/s00063-005-1075-4

7) Sechi A, Fabbro E, Langeveld M, Tullio A, Lachmann R, Mochel F et al. Education and training in adult metabolic medicine: Results of an international survey. JIMD Rep 2019; 49(1): 63-69. e-pub ahead of print 2019/09/10; doi: 10.1002/jmd2.12044

8) Hannah-Shmouni F, Stratakis CA, Sechi A, Langeveld M, Hiwot TG, Tchan MC et al. Subspecialty training in adult inherited metabolic diseases: a call to action for unmet needs. Lancet Diabetes Endocrinol 2019; 7(2): 82-84. e-pub ahead of print 2019/01/27; doi: 10.1016/S2213-8587(18)30369-3

9) Gariani K, Nascimento M, Superti-Furga A, Tran C. Clouds over IMD? Perspectives for inherited metabolic diseases in adults from a retrospective cohort study in two Swiss adult metabolic clinics. Orphanet J Rare Dis 2020; 15(1): 210. e-pub ahead of print 2020/08/20; doi: 10.1186/s13023-020-01471-z

10) van Wegberg AMJ, MacDonald A, Ahring K, Belanger-Quintana $A$, Blau N, Bosch AM et al. The complete European guidelines on phenylketonuria: diagnosis and treatment. Orphanet J Rare Dis 2017; 12(1): 162. e-pub ahead of print 2017/10/14; doi: 10.1186/ s13023-017-0685-2

11) Camp KM, Parisi MA, Acosta PB, Berry GT, Bilder DA, Blau N et al. Phenylketonuria Scientific Review Conference: state of the science and future research needs. Molecular genetics and metabolism 2014; 112(2): 87-122. e-pub ahead of print 2014/03/29; doi: 10.1016/j.ymgme.2014.02.013

12) Ballhausen D, Baumgartner JM, Bonafe L, Fiege B, Kern I, Nuoffer JM. Swiss Metabolic Group: Recommandations pour le traitement de la phenylce-tonurie et de líhyperphenylalaninemie. Paediatrica 2006; 17(2): 14.

13) Linari S, Castaman G. Clinical manifestations and management of Gaucher disease. Clin Cases Miner Bone Metab 2015; 12(2): 157-164. e-pub ahead of print 2015/11/26; doi: 10.11138/ $\mathrm{ccmbm} / 2015.12 .2 .157$

14) Biegstraaten M, Cox TM, Belmatoug N, Berger MG, Collin-Histed T, Vom Dahl S et al. Management goals for type 1 Gaucher disease: An expert consensus document from the European working group on Gaucher disease. Blood Cells Mol Dis 2018; 68: 203-208. e-pub ahead of print 2017/03/10; doi: 10.1016/j. bcmd.2016.10.008

15) Martin MA, Lucia A, Arenas J, Andreu AL. Glycogen Storage Disease Type V. In: Adam MP, Ardinger HH, Pagon RA, Wallace SE, Bean LJH, Stephens K et al. (eds). GeneReviews((R)): Seattle (WA), 1993.

Auf Grund der Beschränkung der Anzahl Referenzen, sind nicht alle Aussagen referenziert. Ein vollständige Liste der Referenzen kann beim Autor eingeholt werden.

\footnotetext{
Autoren

Dr. med. Christel Tran, Centre des Maladies Moléculaires, Service de Médecine Génétique, Centre Hospitalier Universitaire Vaudois, Lausanne

Prof. Dr. med. Andrea Superti-Furga, Centre des Maladies Moléculaires, Service de Médecine Génétique, Centre Hospitalier Universitaire Vaudois, Lausanne PD Dr. med. Michel Hochuli, Universitätsklinik für Diabetologie, Endokrinologie, Ernährungsmedizin und Metabolismus Inselspital, Universitätsspital Bern

Die Autoren haben keine finanziellen oder persönlichen Verbindungen im Zusammenhang mit diesem Beitrag deklariert.
} 